\title{
Article \\ Deletion of a Hand1 lncRNA-Containing Septum Transversum Enhancer Alters lncRNA Expression but Is Not Required for Hand1 Expression
}

\author{
Rajani M. George (D) and Anthony B. Firulli * $D$
}

check for updates

Citation: George, R.M.; Firulli, A.B. Deletion of a Hand1

lncRNA-Containing Septum

Transversum Enhancer Alters lncRNA Expression but Is Not Required for Hand1 Expression. J

Cardiovasc. Dev. Dis. 2021, 8, 50 .

https://doi.org/10.3390/jcdd8050050

Academic Editors: Andy Wessels and Antonio Baldini

Received: 1 April 2021

Accepted: 1 May 2021

Published: 4 May 2021

Publisher's Note: MDPI stays neutral with regard to jurisdictional claims in published maps and institutional affiliations.

Copyright: (C) 2021 by the authors Licensee MDPI, Basel, Switzerland. This article is an open access article distributed under the terms and conditions of the Creative Commons Attribution (CC BY) license (https:// creativecommons.org/licenses/by/ $4.0 /)$
Herman B Wells Center for Pediatric Research, Departments of Pediatrics, Anatomy and Medical and Molecular Genetics, Indiana Medical School, Indianapolis, IN 46202, USA; rmgeorge@iu.edu

* Correspondence: tfirulli@iu.edu; Tel.: +1-317-278-5814

\begin{abstract}
We have previously identified a Hand1 transcriptional enhancer that drives expression within the septum transversum, the origin of the cells that contribute to the epicardium. This enhancer directly overlaps a common exon of a predicted family of long non-coding RNAs (lncRNA) that are specific to mice. To interrogate the necessity of this Hand1 enhancer, as well as the importance of these novel lncRNAs, we deleted the enhancer sequences, including the common exon shared by these lncRNAs, using genome editing. Resultant homozygous Hand1 enhancer mutants $\left(H a n d 1^{\Delta S T / \Delta S T}\right)$ present with no observable phenotype. Assessment of lncRNA expression reveals that Hand1 ${ }^{\Delta S T / \Delta S T}$ mutants effectively eliminate detectable lncRNA expression. Expression analysis within $H a n d 1^{\Delta S T / \Delta S T}$ mutant hearts indicates higher levels of Hand1 than in controls. The generation of Hand1 compound heterozygous mutants with the Hand1 ${ }^{\text {LacZ }}$ null allele (Hand1 ${ }^{\Delta S T / L a c Z}$ ) also did not reveal any observable phenotypes. Together these data indicate that deletion of this Hand1 enhancer and by consequence a family of murine-specific lncRNAs does not impact embryonic development in observable ways.
\end{abstract}

Keywords: HAND1; transcription; heart; epicardium; septum transversum; cardiac morphogenesis; lncRNA

\section{Introduction}

The epicardium, the outer covering of the developing heart is a dynamic structure that contributes to cardiac myofibroblasts, coronary smooth muscle, adipocytes and possibly some coronary endothelium within the mature heart [1]. Epicardial cells originate from an embryonic structure termed the proepicardial organ (PEO), which itself is derived from cells that originate within the anterior portion of the septum transversum (ST) [2-4]. The ST is a transient embryonic structure that arises from a folding of the lateral mesoderm caudal to the developing heart in mammals and gives rise to structures that physically separate the abdominal and thoracic cavities [5]. In mouse E9.0 embryos, cells that originate from the anterior ST undergo epithelial to mesenchymal transition (EMT), move into the PEO, and begin to migrate over the outer surface of the developing heart giving rise to the epicardium. By E10.5 the developing heart is completely covered by epicardial cells. At E12.5, a subset of epicardial cells undergo a successive round of EMT and move into the underlying myocardium and it is these epicardial derived cells which differentiate further into cardiac myofibroblasts, coronary smooth muscle, cardiac adipocytes, and possibly coronary endothelial cells [6-9].

The basic helix loop helix (bHLH) transcription factors HAND1 and HAND2 are required for cardiogenesis and have been implicated in epicardial formation. [10-12]. Lineage trace analysis reveals that Hand1-lineage marks cells within the anterior ST, PEO, epicardium and its secondary EMT derivatives; however, Hand1 expression is only detectable within the anterior ST [13]. Hand2 expression is observed within the PEO and 
epicardium [13]. Deletion of Hand2 using WT1 ERT2Cre [14], which is expressed specifically in the epicardium during development, results in a similar phenotype as the deletion of Hand2 within the Hand1-lineage, malformed coronaries [13].

Interrogation of the conserved non-coding DNA regulatory elements upstream of the Hand1 transcriptional start site has identified an enhancer element that drives LacZ reporter expression within the anterior ST [15]. Interestingly, a family of predicted murine-specific lncRNAs overlaps this ST enhancer. A common exon shared within this lncRNA family resides within the ST enhancer sequences. To determine the function of this Hand1 enhancer and the possible role of these novel predicted lncRNAs in the formation of the PEO and epicardium, we employed CRISPR/Cas9 to delete the ST enhancer and subsequently the lncRNAs. Results show that we obtained 6 Hand $1^{\Delta S T}$ lines of insertion-deletion (indels) which span a range of 1795 to 1786 base pairs of deletion. The largest deletion (1795 bp) was bred to homozygosity-producing viable mice and used for all subsequent experiments. Hand1 expression is observed to be elevated within the heart; however, assessment of PEO, epicardial and epicardial derivative marker gene expression reveal no significant changes supporting the observation that epicardium and epicardial derived structures appear normal. Compound intercross of the Hand1 $1^{\Delta S T}$ with the $H a n d 1^{\text {LacZ }}$ null allele [11] did not introduce any notable phenotypes. Expression analysis confirms that the lncRNA family expression is compromised within $\operatorname{Hand1}^{\Delta S T / \Delta S T}$ embryos. Thus, although this Hand1 enhancer drives ST-specific expression, it is not necessary for the formation of the $\mathrm{PEO}$ or epicardium nor does it reduce expression within the ST, suggesting additional unidentified enhancers can compensate for its loss. Finally, the novel murine-specific lncRNAs, when deleted do not reveal an observable functional role.

\section{Materials and Methods}

\subsection{Mouse Strains and Genotyping}

Hand $1^{\triangle S T}$ mice were generated using CRISPR/Cas9 by the Washington University Genome Engineering (St. Louis, MO, USA) and iPSC core in the FVB background. 6 founder lines were screened for enhancer knockout. Lines with spurious insertions were identified by PCR isolating, TOPO cloning, and sequencing the enhancer region. The largest knockout without artifact (1795 bp deletion) was used for subsequent experiments. For controls, FVB mice were maintained as a separate line for $\mathrm{Hand}^{+/+}$controls. Genotyping was carried out using Southern blots as previously described [16]. BamHI genomic DNA digestion produces an RFLP of $1.6 \mathrm{~kb}$ for the Hand $1{ }^{\Delta \mathrm{ST}}$ allele and $3.6 \mathrm{~kb}$ for the wildtype allele. Homozygous $H a n d 1^{\Delta S T / \Delta S T}$ mice were used for timed matings.

\subsection{Histology}

Embryos were fixed in $4 \%$ paraformaldehyde, dehydrated, embedded, sectioned, and hematoxylin and eosin (H and E) stained as described [16].

\subsection{Cloning}

Southern blot probe for the Hand $1^{\Delta S T}$ allele was amplified and cloned from genomic DNA obtained from FVB mice using Forward 5'-TCGCTGGTTTCTAGCTGTGA- ${ }^{\prime}$ and Reverse $5^{\prime}$-CAGCCCAAATTGCCAGACAC-3' primer sequences. PCR products were cloned into a pCRII-TOPO backbone (ThermoFisher, Waltham, MA, USA). Plasmids were sequenced, digested with EcoRI, gel purified, and radiolabeled with $\alpha^{32} \mathrm{P}$ dCTP using Primea-Gene Labeling System (Promega, Madison, WI, USA) to use as probe for Southern blots.

\subsection{In-Situ Hybridization}

Section in-situ hybridizations (ISH) were performed on $10-\mu \mathrm{m}$ paraffin sections as described previously $[16,17]$. Whole mount in-situ hybridizations were performed using E10.5 day embryos as described previously [16]. Antisense digoxygenin-labeled riboprobes were synthesized using T7, T3, or SP6 polymerases (Promega) and DIG-Labeling Mix 
(Roche, Basel, Switzerland) using the following plasmid templates: Hand1, Tbx18, Tcf21, and Postn.

\subsection{Quantitative Real Time PCR}

Total RNA was isolated from E9.5 hearts or torsos not including the heart, or E10.5 hearts alone using the High Pure RNA Isolation Kit (Roche). RNA was used to synthesize cDNA using the High-Capacity cDNA Reverse Transcription Kit (Applied Biosystems, Waltham, MA, USA). For qRT-PCR, cDNA was amplified using TaqMan Probe-Based Gene Expression Assays (Applied Biosystems) to quantify Hand1, Tcf21, and Tbx18 expression in E9.5 hearts and torsos. Custom primers specific to ST enhancer sequence and putative lncRNAs were synthesized by Integrated DNA Technologies (IDT) P1[F]5'TGCCGCCGCACGTCTCTAAT-3' P1[R]5'-ATCCACAGGGCTGCCCTATC-3' P2[F]5'AGCTCCTTGAGGCCAGGGAG-3' P2[R]5'-TAATAGGCAGGAGGTCAATCCCTC-3' P3[F]5' AGCAGAGTCTCACTGAACCATACTCCACC-3' P3[R]5'-AGAGTTGGTTGACCATGTG AGTTATGTGTGAACC-3' ${ }^{\prime}$. cDNA in E10.5 day hearts was assayed using PowerUp SybrGreen Master Mix (ThermoFisher). qRT-PCR reactions were run on the QuantStudio 3 Real-Time PCR System (ThermoFisher). Normalization to Glyceraldehyde 3-phosphate dehydrogenase (GAPDH) was used to determine relative gene expression and statistical analysis was automatically applied by the instrument software. Significance of qRT-PCR results was determined by a two-tailed student's t-test followed by post hoc BenjaminiHochberg FDR correction as automatically calculated by the QuantStudio 3 qRT-PCR thermal cycler software analysis package.

\subsection{ATAC-Seq}

Assay for Transposase-Accessible Chromatin by sequencing was done as previously described [18]. In brief, timed matings were used to generate E10.5 embryos. Hearts were excised in PBS, and single-cell suspension was obtained using the Wheaton tight douncer, and 50,000 cells collected. Cells were lysed in $10 \mathrm{mM}$ Tris pH 7.4, $10 \mathrm{mM} \mathrm{NaCl}, 3 \mathrm{mM} \mathrm{MgCl} 2$ and $0.1 \%$ IGEPAL CA- 630 for $5 \mathrm{~min}$ on ice. Nuclei were pelleted and $50 \mu \mathrm{L}$ tagmentation reaction and subsequent sequencing carried out as per manufacturer's recommendations (Illumina, San Diego, CA, USA).

\section{Results}

\subsection{CRISPR/Cas9 Mediated Deletion of the ST Enhancer}

Previous work has identified a putative cis-regulatory enhancer element $34 \mathrm{~kb}$ upstream of transcriptional start site of Hand1 coding sequence marking the ST at embryonic day (E)10.5 (Figure 1A, red box) [15]. ATAC-seq (Assay for Transposase-Accessible Chromatin by sequencing) in E10.5 hearts shows accessible chromatin at this genomic locus at this time point. In order to further characterize this enhancer, we used CRISPR/Cas9 mediated deletion of the $1.8 \mathrm{~kb}$ element located at genomic region chr11:57,678,478-57,680,329 (mm9). Guide RNAs (Figure 1A, green bars) were designed for this region and 6 founder lines were generated. These lines were screened for deletion size, and presence of spurious insertions at the locus by PCR amplification, TOPO cloning of insert, and sequencing. The largest deletion without artifact was selected for further breeding. This line, with a $1795 \mathrm{bp}$ deletion, generated viable, phenotypically normal, homozygous $H a n d 1^{\Delta S T / \Delta S T}$ mice as determined by Southern blotting (Figure 1B). These mice were maintained on an FVB background and control Hand1 ${ }^{+/+}$mice were generated by breeding wildtype FVB mice. Hand $1^{+/+}$wildtype allele was detected as a $3.6 \mathrm{~kb}$ fragment, and the Hand $1^{\Delta S T / \Delta S T}$ enhancer knockout allele was a $1.6 \mathrm{~kb}$ fragment on the Southern blot (Figure 1B). Hand $1^{\Delta S T / \Delta S T}$ mice were bred to homozygosity and did not show any apparent phenotype. Neonates were obtained at expected ratios and histological analysis of post-natal day 1 Hand $1^{\Delta S T / \Delta S T}$ hearts did not show any defects when compared to Hand1 ${ }^{+/+}$controls (Figure 1C). ClustalW alignment across mammals, comparing mouse sequence to human, rat, cow, and dog shows regions of conservation in consensus transcription factor binding sites (Figure 1D). 
A

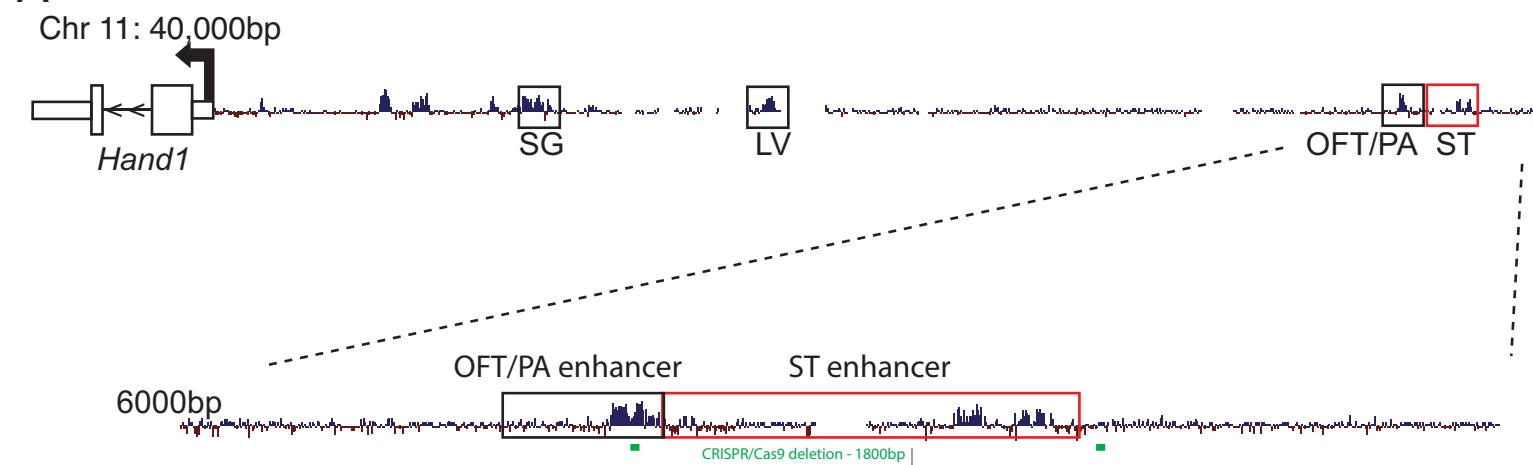

ATAC E10.5 Heart

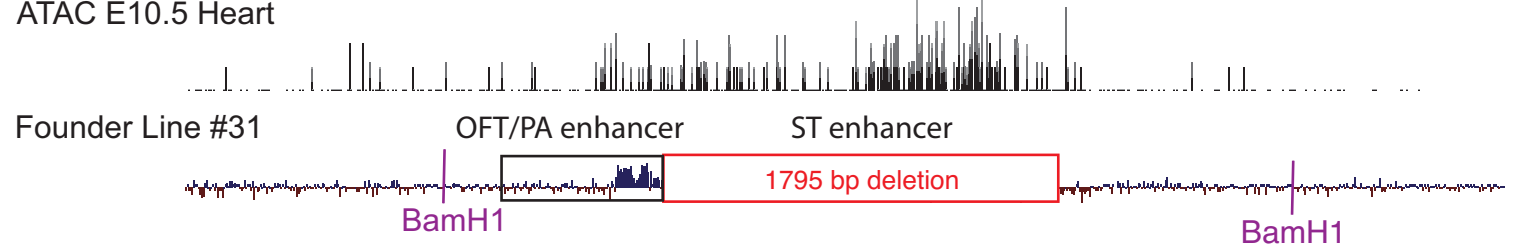

B

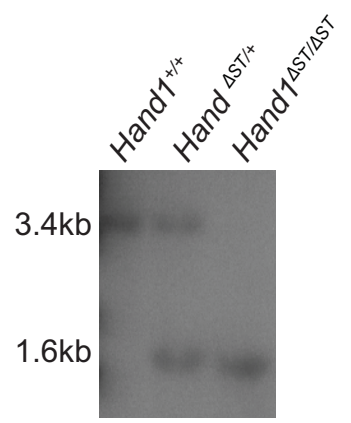

\section{C}
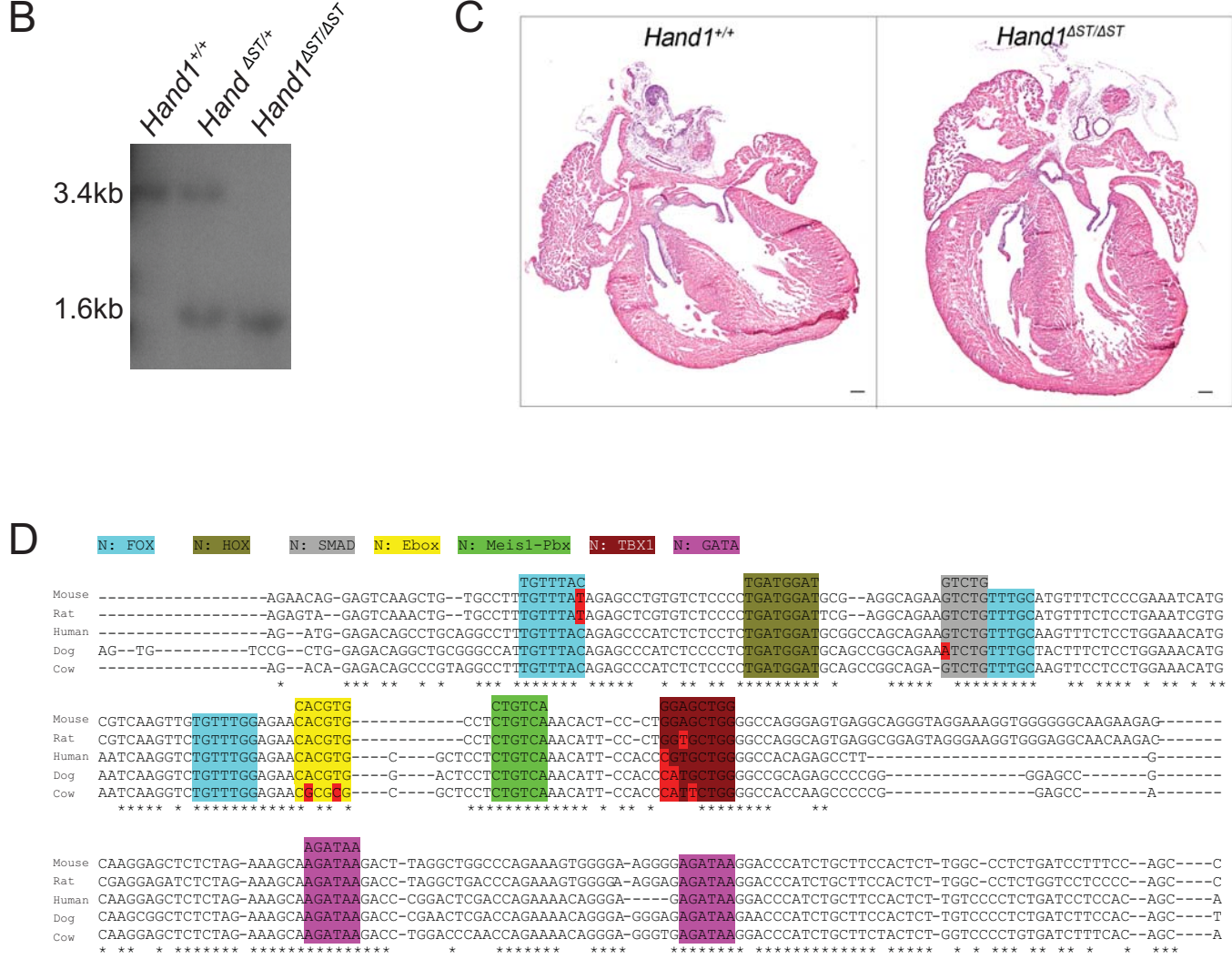

Figure 1. CRISPR/Cas9 mediated deletion of ST enhancer. (A) Mammalian conservation track at the mouse Hand1 locus. Conserved non-coding regions with spatial and temporal specific activity are boxed. ST enhancer is boxed in red. Close up region of OFT and ST enhancer are indicated with overlying ATAC-seq from E10.5 hearts. Bars in green represent guide RNA sequences designed for CRISPR/Cas9 mediated deletion of ST enhancer. The largest deletion line used for further analysis with 1795 bp deletion is shown. Representative BamH1 digest sites for Southern blot analysis are indicated. Location of Southern blot probe is indicated. (B) Southern blot analysis for genotyping Hand1 ${ }^{\Delta S T / \Delta S T}$. Wildtype Hand1 ${ }^{+/+}$, Heterozygote $H a n d 1^{\Delta S T /+}$, and Homozygote Hand1 ${ }^{\Delta S T / \Delta S T}$ are shown. The Hand1 ${ }^{+}$allele is $3.6 \mathrm{~kb}$ in length and the Hand1 ${ }^{\Delta S T}$ allele is $1.6 \mathrm{~kb}$ in length. (C) Post-natal day 1 hearts from $H a n d 1^{+/+} H a n d 1^{\Delta S T / \Delta S T}$ mice. $10 \mu \mathrm{m}$ wax sections are stained with hemotoxylin and eosin. Scale bar $100 \mu \mathrm{m}$. (D) ClustalW pile up for core conserved region of ST enhancer comparing mouse, rat, human, cow, and dog sequences. Consensus DNA sequence for transcription factor binding sites is indicated. LV, left ventricle; OFT, outflow tract; PA, pharyngeal arches; SG, sympathetic ganglia; ST, septum transversum; ATAC-seq, assay for transposase-accessible chromatin using sequencing. 


\subsection{Loss of a Hand1 Allele over the ST Enhancer Knockout Doesnot Lead to Embryonic Lethality}

Since Hand $1^{\Delta S T / \Delta S T}$ mice do not demonstrate any observable phenotype, we crossed homozygous Hand1 ${ }^{\Delta S T / \Delta S T}$ to the Hand1 ${ }^{\text {LacZ/+ }}$ mouse [11] to determine if loss of one functional Hand1 allele resulted in a more deleterious phenotype. Embryos from the resulting cross were subjected to whole mount in-situ hybridization (WISH) at E10.5 to determine spatial or qualitative changes in Hand1 gene expression levels. Comparison of Hand $1^{+/+}$ controls to Hand1 $1^{\Delta S T / L a c Z}$ or Hand1 ${ }^{\Delta S T / \Delta S T}$ embryos showed no appreciable difference in Hand 1 expression (Figure 2A-C). Since the OFT/PA enhancer is directly $3^{\prime}$ of the ST enhancer [15], we carefully examined PA expression of Hand1 within Hand1 ${ }^{\Delta S T / \Delta S T}$ embryos. Results reveal that Hand1 PA expression is maintained within Hand1 ${ }^{\Delta S T / \Delta S T}$ embryos (arrow, Figure 2B) as well as within Hand $1^{\Delta S T / L a c Z}$ embryos (arrow, Figure 2C). We also examined expression within the umbilical region just caudal to the heart and observed no significant changes in Hand1 expression (arrows, Figure $2 \mathrm{~A}^{\prime}-\mathrm{C}^{\prime}$ ) Analysis of mendelian ratios from breeding Hand1 ${ }^{\Delta S T / \Delta S T}$ and Hand $1^{\text {LacZ/+ }}$ mice $(n=40)$ show Hand $1^{\Delta S T / L a c Z}$ pups are encountered at expected frequency when genotyped at postnatal day 10, and these mice are viable and fertile (Figure 2D).
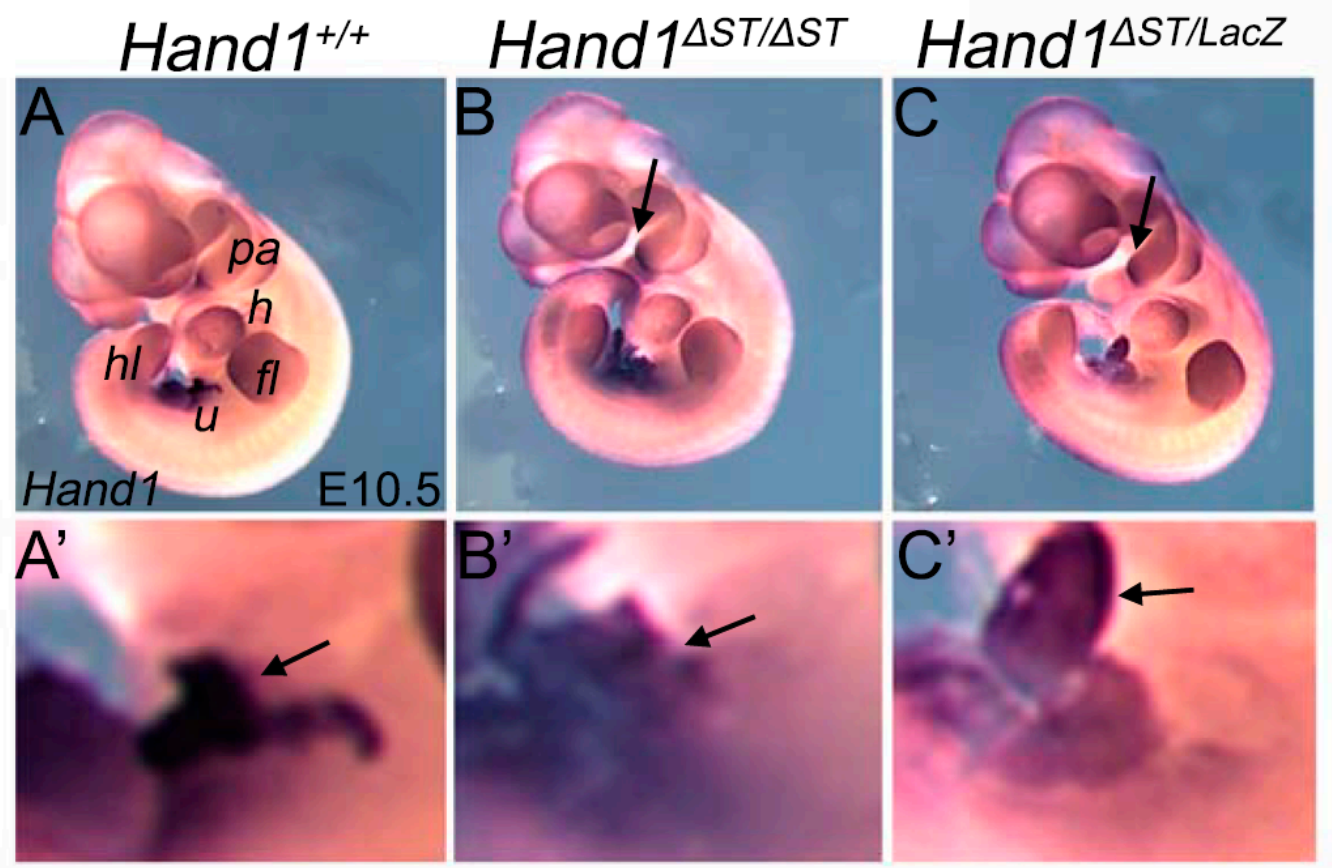

D

\begin{tabular}{|lcc|}
\hline Genotype & Expected $(n=40)$ & Recovered \\
\hline Hand ${ }^{+/ \Delta S T}$ & 20 & 21 \\
\hline Hand $1^{\Delta S T / L a c Z}$ & 20 & 19 \\
\hline
\end{tabular}

Figure 2. Loss of a Hand1 Allele over the ST Enhancer knockout does not lead to embryonic lethality. (A-C) Whole mount in-situ hybridization (WISH) at E9.5 for Hand1 mRNA in Hand1 ${ }^{+/+}$, Hand $1^{\Delta \mathrm{ST} / \Delta \mathrm{ST}}$, and Hand $1^{\Delta \mathrm{ST} / \mathrm{LacZ}}$ embryos showing no qualitative or spatial change in Hand 1 mRNA levels. Arrows indicating pharyngeal arch expression of Hand1 in Hand1 ${ }^{\Delta \mathrm{ST} / \Delta \mathrm{ST}}$ and Hand1 ${ }^{\Delta \mathrm{ST}} /$ LacZ embryos. $N=5$ embryos for all genotypes. pa, pharyngeal arches; $\mathrm{h}$, heart; fl, forelimb; hl, hindlimb; $u$, umbilicus. (A'-C') Umbilicus region expression of Hand1 in WISH embryos. (D) Table showing expected and recovered genotypes at postnatal day 10 from Hand $1^{\Delta \mathrm{ST} / \Delta \mathrm{ST}}$ and Hand $1^{\mathrm{LacZ} /+}$ parental crosses. 


\subsection{ST and PEO In Hand1 ${ }^{\Delta S T / \Delta S T}$ Embryo}

In order to carefully identify the ST and PEO within Hand $1^{\Delta S T / \Delta S T}$ embryos, we interrogated expression of transcription factor Tbx18. Tbx18 expression marks the ST, the PEO, and epicardial cells laminating over the cardiac surface at E9.5 (Figure 3A,E,I) [19,20]. Hand1 is expressed within the ST but not the PEO (Figure 3C,G) [13]. To test if ST and/or PEO development within Hand $1^{\Delta S T / \Delta S T}$ embryos is affected by loss of the ST enhancer, E9.5 embryos were transverse sectioned and serial sections used for ISH to detect Hand1 and Tbx18 mRNA. Analysis of mRNA expression in Hand1 ${ }^{\Delta S T / \Delta S T}$ embryos shows Tbx18 expression within the ST of Hand $1^{\Delta S T / \Delta S T}$ embryos is similar to that observed in Hand1 ${ }^{+/+}$ controls (Figure 3A,B). Surprisingly, Hand1 expression within the ST appears indistinguishable when comparing Hand $1^{\Delta S T / \Delta S T}$ and $H a n d 1^{+/+}$embryos (Figure 3C,D), suggesting that Hand1 expression within the ST is regulated by redundant enhancers.

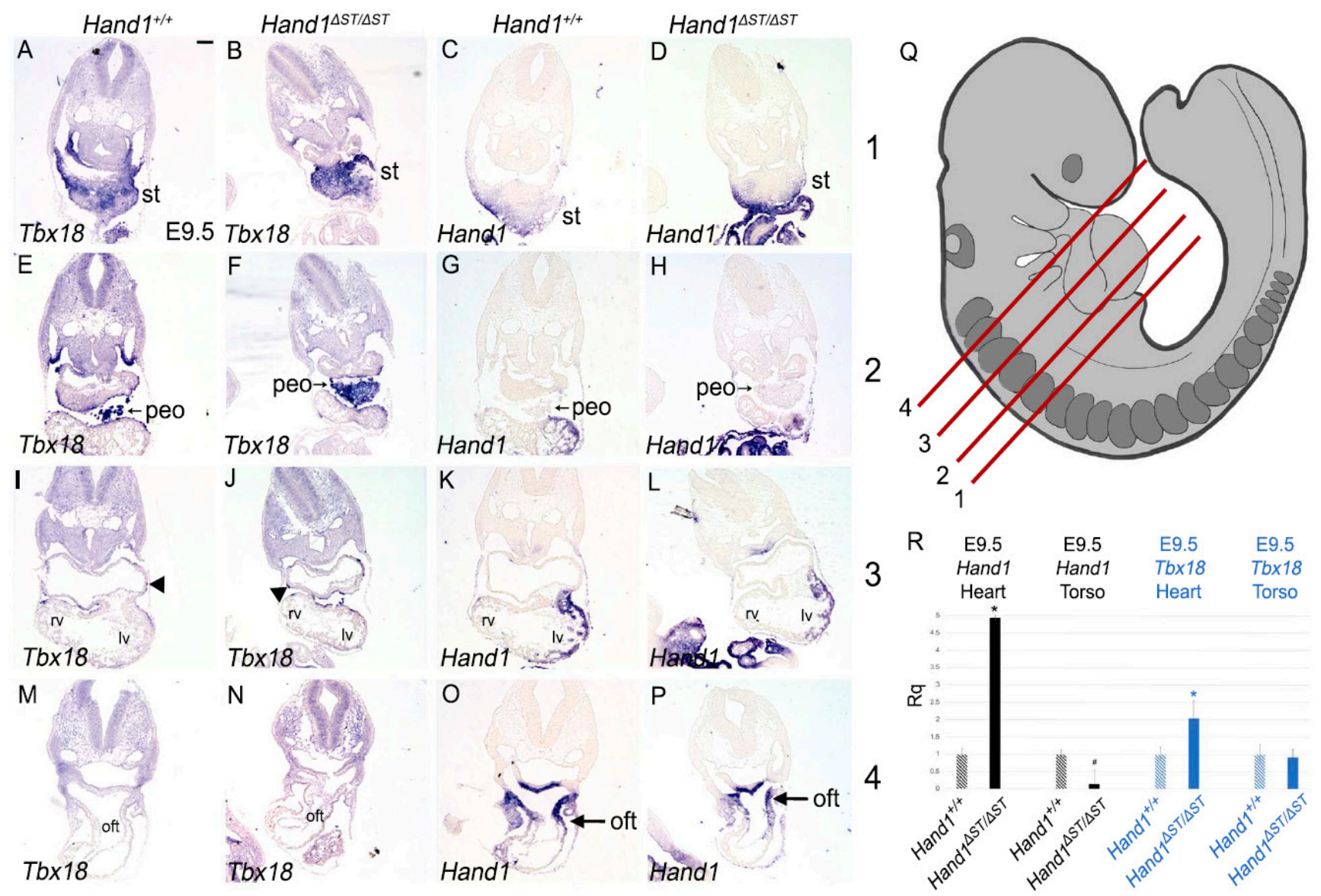

Figure 3. ST And PEO In Hand1 ${ }^{\Delta S T / \Delta S T}$ Embryo. (A-P) Section in-situ hybridization at E9.5 for Tbx18 and Hand1 mRNA in $H a n d 1^{+/+}$and Hand $1^{\Delta S T / \Delta S T}$ embryos. Arrowheads indicate Tbx18 expressing cells migrating over the heart to form the epicardium. Arrows indicate Hand1 expression in the outflow tract. st, septum transversum; peo, proepicardial organ; rv, right ventricle; lv, left ventricle; oft, outflow tract. $n=6$ for all genotypes. Scale bar 100 $\mathrm{mm}$. (Q) Representation of E9.5 embryo showing planes of section indicated at rows: 1 (st), 2 (peo), 3 (four chamber heart), 4 (developing outflow tract). (R) qRT-PCR at E9.5 for Hand1 and Tbx18 expression in heart, and torso (dissected to exclude the heart, but with PEO and ST intact) in Hand $1^{+/+}$and Hand $1^{\Delta S T / \Delta S T}$ embryos. $n=9$ embryos, pooled. Significant increase in Hand $1{ }^{*}$ indicates $p \leq 0.01$, \# indicates $p \leq 0.05)$ and Tbx18 (* indicates $p \leq 0.01$ ) are observed. Error bars represent the high and low range of replicate cycle reads within each primer set. 
Cells within the PEO are identified by Tbx18 expression and their characteristic rounded cellular morphology at E9.5 as they migrate to envelop the developing heart (Figure 3E) [21]. Hand1 expression is not observed within the PEO, as has been previously reported (Figure 3G) [13]. In Hand1 ${ }^{\Delta S T / \Delta S T}$ embryos at a similar plane of section, we observe PEO cells that express Tbx18 (Figure 3F). In both Hand1 ${ }^{+/+}$control and Hand1 ${ }^{\Delta S T / \Delta S T}$ embryos, $T b x 18$ expressing cells are observed laminating over the heart to give rise to the epicardium (arrowheads, Figure 3IJJ). Hand1 expression within the left ventricle (LV) and outflow tract (OFT) appear unchanged within Hand1 ${ }^{\Delta S T / \Delta S T}$ embryos compared to Hand $1^{+/+}$controls (Figure $3 \mathrm{~K}, \mathrm{~L}, \mathrm{O}, \mathrm{P}$ arrow). In order to quantify any changes in gene expression, $\mathrm{Hand}_{1}{ }^{+/+}$and $\mathrm{Hand} 1^{\Delta S T / \Delta S T}$ embryos at E9.5 are dissected to separate the heart from the torso and are pooled for qRTPCR using Taqman probes against Hand1 and Tbx18. Surprisingly, results show that Hand1 expression within $\operatorname{Hand}^{\Delta S T / \Delta S T}$ hearts is significantly upregulated (4.935 fold, $p=0.001$ ) as compared to Hand $1^{+/+}$controls (Figure 3R). Hand1 expression levels within the torso, excluding the heart, but including the ST and PEO, are significantly decreased in Hand $1^{\Delta S T / \Delta S T}$ compared to Hand $1^{+/+}(0.143$ fold, $p=0.034)$ (Figure 3R). Tbx18 expression in E9.5 hearts is increased by a modest but significant (2.026 fold, $p=0.001$ ) level within Hand $1^{\Delta S T / \Delta S T}$ compared to Hand1 ${ }^{+/+}$controls (Figure 3R). Tbx18 expression within Hand $1^{+/+}$and Hand $1^{\Delta S T / \Delta S T}$ torsos remains unchanged (0.909 fold, $p=0.368)$ (Figure 3R).

\subsection{Expression of Hand2 in Hand1 ${ }^{\Delta S T / \Delta S T}$ Embryos}

Hand1 and Hand2 are expressed in overlapping domains during heart development [12]. Previous work has also suggested functional redundancy in function of these factors $[13,22]$. To determine if loss of the ST enhancer results in changes in Hand2 expression, E9.5 embryos were sectioned and Hand $1^{+/+}$and Hand $1^{\Delta S T / \Delta S T}$ embryos were compared (Figure 4). As previously reported, Hand2 is robustly expressed in the ST (Figure 4A,C) and PEO (Figure 4E,G) [13]. Hand2 domain of expression in Hand $1^{\Delta S T / \Delta S T}$ embryos compared to Hand1 $1^{+/+}$in ST (Figure 4B,D) and PEO (Figure 4F,H) appears unchanged. In the four-chambered heart, Hand2 expression appears unchanged between Hand1 $1^{+/+}$and Hand $1^{\Delta S T / \Delta S T}$ embryos (Figure $4 \mathrm{~K}, \mathrm{~L}$ ). Loss of the ST enhancer in Hand1 ${ }^{\Delta S T / \Delta S T}$ embryos does not appear to change the domain or qualitative level of Hand2 expression in the ST or its derivatives. qRTPCR analysis using Taqman probes against Hand2 in pooled E9.5 embryos show Hand2 expression levels as trending lower but not significantly different within Hand $1^{\Delta S T / \Delta S T}$ compared to Hand $1^{+/+}$hearts $(0.657$ fold, $p=0.083$ ) (Figure $4 \mathrm{M}$ ). Hand 2 expression within the torso, similar to Hand1, is significantly decreased ( 0.390 fold, $p=0.021)$ in Hand $1^{\Delta S T / \Delta S T}$ compared to Hand $1^{+/+}$embryos (Figure $4 \mathrm{M}$ ).

\subsection{Development of the Epicardium Is Unaffected In Hand ${ }^{\Delta S T / \Delta S T}$ Embryo}

During heart development, the ST is the source of cells that gives rise to the epicardium [1]. By E13.5, epicardial cells cover the four-chambered heart and are marked by expression of Tcf21 [23]. To determine if loss of the ST enhancer results in changes in epicardial development, E13.5 Hand1 ${ }^{+/+}$and Hand1 ${ }^{\Delta S T / \Delta S T}$ embryos were sectioned.

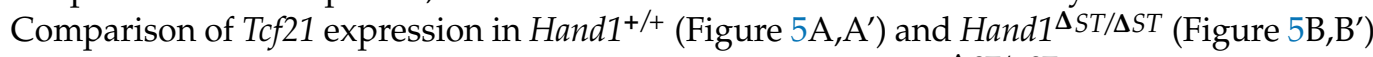
epicardium suggests that epicardial development in $\operatorname{Hand1}^{\Delta S T / \Delta S T}$ embryos is normal. Higher power views of Tcf21 expression at the epicardial surface (red square Figure 4A,B) reveal infiltration of Tcf21 positive epicardial cells within myocardium indicating the expected secondary EMT observed in normal development (Figure $\left.5 \mathrm{~A}^{\prime}, \mathrm{B}^{\prime}\right)$. qRT-PCR analysis for Tcf21 using Taqman probes in E13.5 hearts suggests a modest but significant increase (1.626 fold, $p=0.005)$ in Tcf21 transcript in Hand1 ${ }^{\Delta S T / \Delta S T}$ hearts compared to Hand1 ${ }^{+/+}$ controls (Figure 5E). 

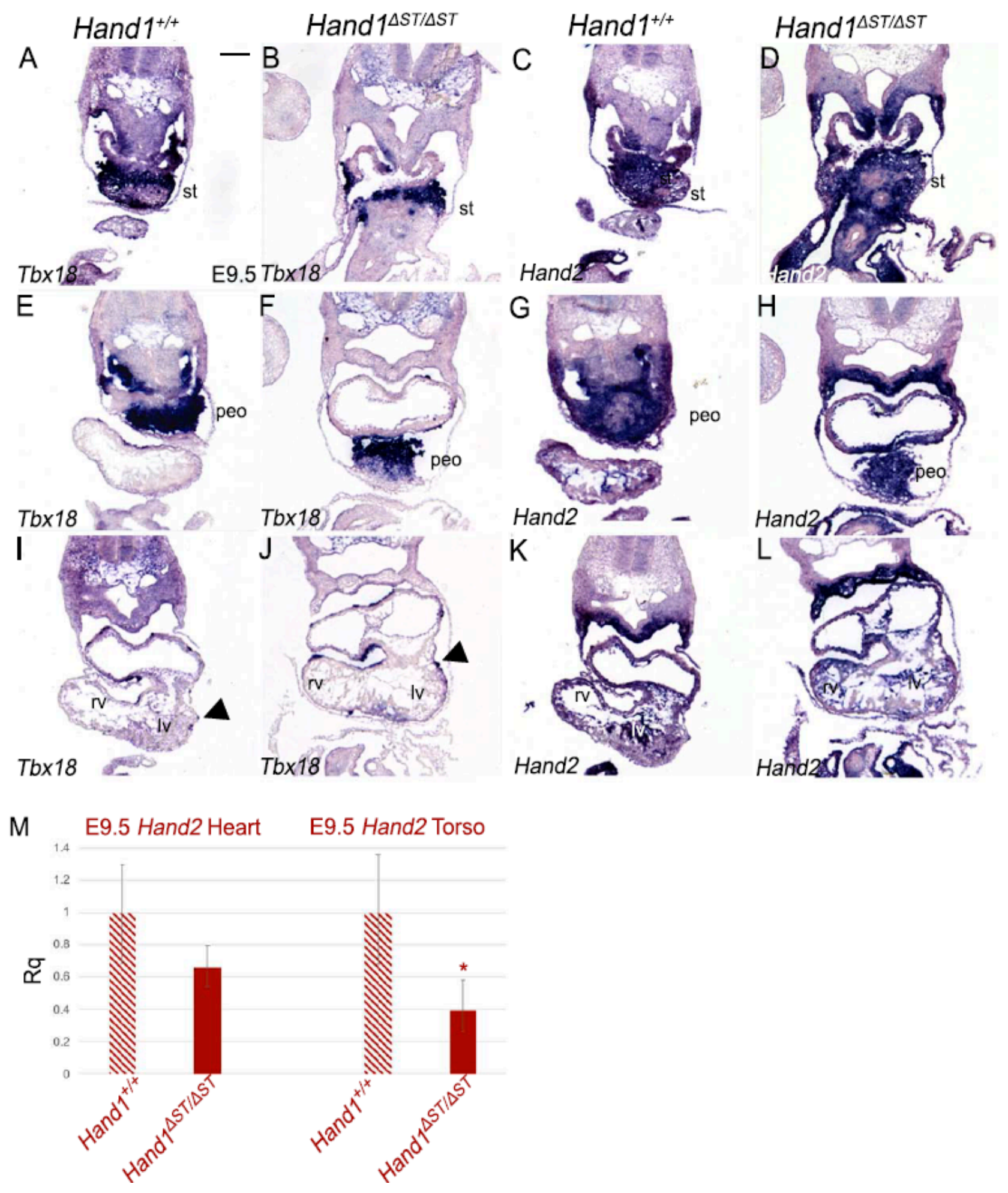

Figure 4. Hand2 expression in Hand1 ${ }^{\Delta S T / \Delta S T}$ Embryos. (A-L) Section in-situ hybridization at E9.5 for Tbx18 and Hand2 mRNA in $H a n d 1^{+/+}$and Hand $1^{\Delta S T / \Delta S T}$ embryos. Arrowheads indicate Tbx18 expressing cells migrating over the heart to form the epicardium. st, septum transversum; peo, proepicardial organ; rv, right ventricle; lv, left ventricle. $n=3$ for all genotypes. Scale bar $100 \mu \mathrm{m}$. (M) qRT-PCR at E9.5 for Hand2 expression in heart, and torso (dissected to exclude the heart, but with PEO and ST intact) in Hand $1^{+/+}$and Hand $1^{\Delta S T / \Delta S T}$ embryos ( ${ }^{*}$ indicates $\left.p \leq 0.01\right) . n=9$ embryos, pooled. Error bars represent the high and low range of replicate cycle reads within each primer set. 


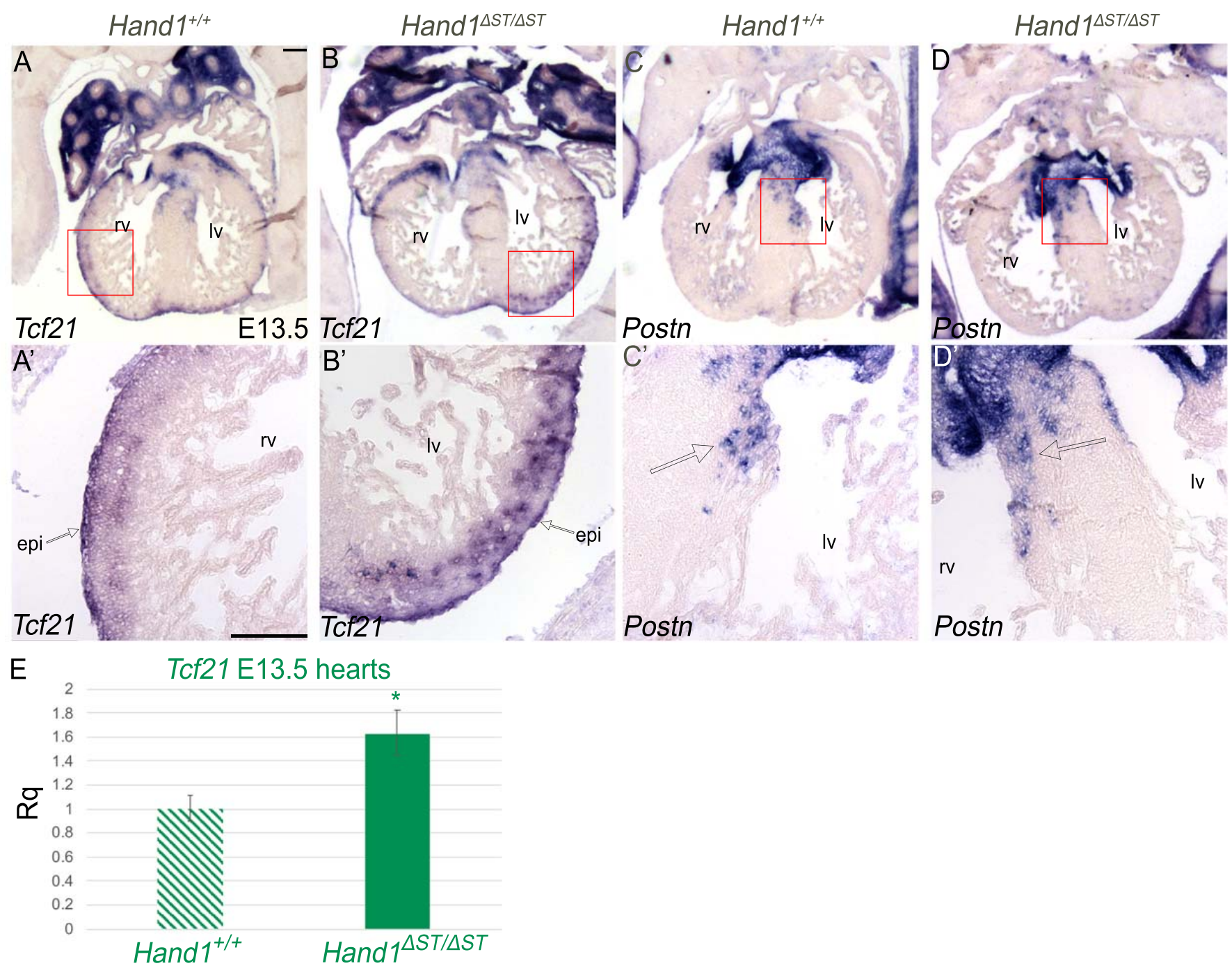

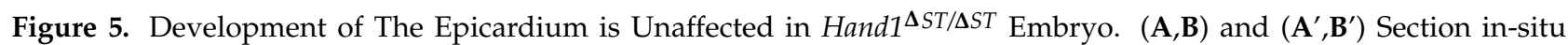
hybridization at E13.5 Hand1 ${ }^{+/+}$and Hand1 ${ }^{\triangle S T / \Delta S T}$ embryos for Tcf21 showing four chamber view and at higher magnification. Arrow indicating epicardium. $(\mathbf{C}, \mathbf{D})$ and $\left(\mathbf{C}^{\prime}, \mathbf{D}^{\prime}\right)$ Section in-situ hybridization at E13.5 Hand1 ${ }^{+/+}$and Hand1 ${ }^{\Delta S T / \Delta S T}$ embryos for Periostin (Postn) showing four chamber view and at higher magnification. Arrow indicating cardiac fibroblasts. rv, right ventricle; lv, left ventricle; epi, epicardium. Scale bar $100 \mu \mathrm{m}$. (E) qRT-PCR at E13.5 for Tcf21 in Hand1 ${ }^{+/+}$and Hand1 $1^{\Delta S T / \Delta S T}$ hearts. Significant increase in Tcf21 ( ${ }^{*}$ indicates $\left.p \leq 0.01\right)$ is observed. Error bars represent the high and low range of replicate cycle reads within each primer set.

Epicardial cells undergo secondary EMT to give rise to epicardial derived cells (EPDCs) that move into the myocardium and eventually differentiate into cardiac fibroblasts that contribute to the developing valves [7]. Periostin (Postn) is expressed by these EPDCs [24]. In order to confirm that loss of ST enhancer appears not to affect EPDC development, section ISH was carried out at E13.5 to examine Postn expression within $H a n d 1^{+/+}$and $H a n d 1^{\Delta S T / \Delta S T}$ embryos (Figure 5C,D). Comparison of $H a n d 1^{+/+}$and Hand $1^{\Delta S T / \Delta S T}$ embryos (Figure $5 \mathrm{C}^{\prime}, \mathrm{D}^{\prime}$ respectively) suggests an increased region of Postn expression in Hand1 ${ }^{\Delta S T / \Delta S T}$ embryos. Given that Tcf21 expression is upregulated within Hand $1^{\Delta S T / \Delta S T}$ embryos along with the increased expression of Postn, our data suggests an expansion of EPDCs in Hand1 ${ }^{\Delta S T / \Delta S T}$ embryos.

\subsection{Loss of Putative LncRNA Family Does Not Affect Heart Development}

The mouse Hand1 locus contains 6 putative lncRNAs (X1-X6) predicted by Gnomon (NCBI eukaryotic gene prediction tool) to be upstream of the Hand1 transcriptional start 
site, that transcribe in the same direction as Hand1, and partially overlap with the ST enhancer (Figure 6A, red box). Primers designed internally to a common exon shared by all 6 of the putative lncRNAs (Figure 6B, P1, blue) detect lncRNA transcript within E10.5 $H a n d 1^{+/+}$hearts, with no expression observed in Hand $1^{\Delta S T / \Delta S T}$ hearts (Figure 6C). Primers that are intron spanning (Figure 6B, P2, magenta) also reveal lncRNA expression within E10.5 $\mathrm{Hand1}^{+/+}$hearts but fail to detect expression within Hand1 ${ }^{\Delta S T / \Delta S T}$ hearts (Figure 6C). Loss of the common exon in Hand $1^{\Delta S T / \Delta S T}$ hearts leads to loss of downstream exons as determined by qRT using primers against the last two exons of X4, X5, X6 (Figure 6B, P3, purple) indicate that loss of the common exon leads to loss of lncRNA transcript as detected by qPCR in Hand1 ${ }^{\Delta S T / \Delta S T}$ E10.5 hearts (Figure $6 \mathrm{C}$ ).

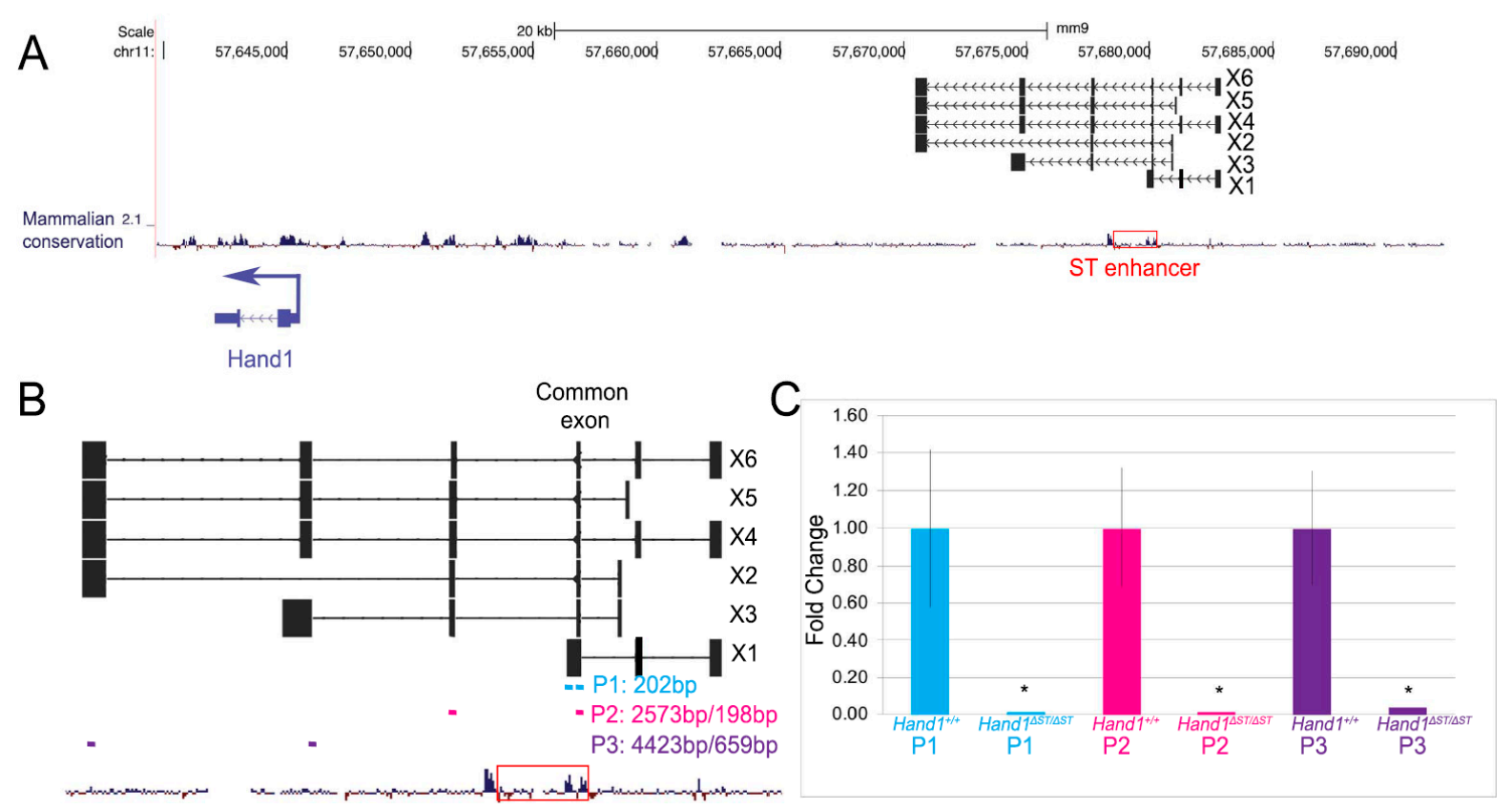

Figure 6. Loss of Putative LncRNA Family does not Affect Heart Development. (A) Schematic showing predicted lncRNAs X1, X2, X3, X4, X5, X6 upstream of Hand1 coding region. Red box indicating ST enhancer deletion. (B) Schematic showing lncRNA overlaid with ST enhancer. Common exon shared between all lncRNAs that overlaps with ST enhancer is marked. Two primer sets designed to detect lncs P1 (blue, 202 bp lncRNA product size), P2 (magenta, 2573 bp genomic product size, 198 bp lncRNA product size), P3 (purple, 4423 bp genomic product size, 659 bp lncRNA product size) are marked. (C) qRT-PCR results to detect lncRNAs in E10.5 Hand1 ${ }^{+/+}$and Hand1 ${ }^{\Delta S T / \Delta S T}$ embryos. ( ${ }^{*}$ indicates $\left.p \leq 0.05\right)$. Error bars indicate standard error.

\section{Discussion}

Hand1 embryonic expression is regulated by a number of tissue-specific enhancers driving tissue-specific expression within post-migratory neural crest cells (NCC) within the sympathetic ganglia, the myocardium of the LV, and within the post-migratory NCC that populate the lower jaw and cardiac OFT [15,22,25]. Hand1 expression is also observed within the ST of the developing embryo [13] and using a Cre recombinase Hand1 knock-in allele [26] to delete Hand2 reveals cardiac phenotypes of epicardial origin [13]. Transgenic reporter analysis identifies a conserved non-coding region that drives expression within the ST [15]. In this study, we delete this ST enhancer to determine its role in regulating Hand1 expression within the ST and its role in PEO and epicardial formation.

Results show clearly that $H a n d 1^{\Delta S T / \Delta S T}$ mice are viable and without any discernable phenotypes. Moreover, Hand $1^{\Delta S T / L a c Z}$ compound heterozygotes are also viable and without any discernable phenotypes. Given that Hand1 expression within the developing ST does not appear changed, we conclude that it is likely that a secondary ST enhancer, as yet unidentified, compensates in Hand1 ${ }^{\Delta S T / \Delta S T}$ mice. It is possible that the Hand1 OFT/PA 
enhancer that lies just $3^{\prime}$ to the ST enhancer might also contribute to Hand1 ST expression; however, previous transgenic reporter analysis does not support this possibility [15]. Interrogation of gene expression within Hand1 $1^{\Delta S T / \Delta S T}$ embryos for markers of the ST and PEO (Tbx18), the epicardium (Tcf21), and established EPDCs (Postn) reveal no change in expression patterns supporting the lack of phenotype observed in these mice.

The ST and PEO, are incompletely marked by Hand1 (Figure 3C) [13], suggesting that non-Hand1 expressing cells might be sufficient to drive ST maturation during development. Additionally, non-PEO origin cells are known to contribute to the epicardium [27]. This heterogeneity of the PEO, and the non-PEO origin of the epicardium could explain a lack of phenotype in the ST enhancer knockout mice; however, given that Hand1 expression patterns are not visibly changed, it is more likely that there is compensation for Hand1 expression from a redundant enhancer.

One curious observation made in $H a n d 1^{\Delta S T / \Delta S T}$ embryos is that we observe a significant increase in Hand1 cardiac expression. This could be the result of removing negative myocardial transcriptional inputs or the repositioning of Hand1 OFT and LV enhancers within the open chromatin such that these enhancers become more efficient. The lack of phenotype in $H a n d 1^{\Delta S T / \Delta S T}$ embryos might also be due to the decrease in Hand2 expression seen in Hand $1^{\Delta S T / \Delta S T}$ hearts (Figure $4 \mathrm{M}$ ), suggesting a feedback regulatory mechanism between Hand1 and Hand2 expression levels in vivo. This compensatory mechanism might be heart-specific, as both Hand1 and Hand2 are significantly downregulated in the torsos within Hand $1^{\Delta S T / \Delta S T}$ embryos. What is clear is that the increase in Hand1 cardiac transcripts does not result in observable phenotype, suggesting that there is broad toleration for different concentrations of Hand1 transcript and/or that established mechanisms of post-translational regulation of HAND1 protein can mitigate deleterious gain-of-function phenotypes [28-30].

Finally, we show that a murine-specific predicted lncRNA family is actually expressed during embryonic development and a common IncRNA exon resides within the Hand1 ST enhancer (Figure 6). The observed increase in Hand1 expression within Hand $1^{\Delta S T / \Delta S T}$ hearts suggests that these lncRNAs might repress Hand1. Results show that deletion of the ST enhancer leads to a loss of expression of this novel lncRNA family; however, no phenotype is observed. Recent work from other groups has shown deletion of cardiacspecific lncRNAs is not required for embryo viability. Given many of these identified IncRNAs are not well conserved through evolution may reflect subtle roles in transcription, mRNA processing, or other molecular mechanisms that are species-specific [31].

Taken together, we conclude from this data that the Hand1 ST enhancer is sufficient to drive ST expression during mouse embryogenesis [15] but is not necessary to maintain sufficient Hand1 expression within the ST suggesting the existence of a redundant Hand1 ST enhancer capable of driving normal epicardial development.

Author Contributions: Conceptualization, R.M.G. and A.B.F.; formal analysis, R.M.G.; investigation, R.M.G.; writing—original draft preparation, R.M.G.; writing—review and editing, R.M.G. and A.B.F.; supervision, A.B.F.; funding acquisition, R.M.G. and A.B.F. All authors have read and agreed to the published version of the manuscript.

Funding: This research was funded by the National Institutes of Health to ABF (P01 HL134599-05 1R01DE029091-01, and R01 HL145060-03) and the American Heart Association to RMG (19POST34381038). Infrastructural support at the Herman B. Wells Center for Pediatric Research is in part supported by the generosity of the Riley Children's Foundation, Division of Pediatric Cardiology, and the Carrolton Buehl McCulloch Chair of Pediatrics.

Institutional Review Board Statement: The study was conducted according to the guidelines of the Declaration of Helsinki, and approved by the Institutional Review Board (or Ethics Committee) of Indiana University School of Medicine (protocol 20090 approved on 4 September 2020).

Acknowledgments: We thank Danny Carney for technical assistance. We thank Beth Firulli for valuable discussion.

Conflicts of Interest: The authors declare no conflict of interest. 


\section{References}

1. Simoes, C.F.; Riley, P.R. The ontogeny, activation and function of the epicardium during heart development and regeneration. Development 2018, 145. [CrossRef]

2. Ho, E.; Shimada, Y. Formation of the epicardium studied with the scanning electron microscope. Dev. Biol. 1978, 66, 579-585. [CrossRef]

3. Viragh, S.; Challice, C.E. The origin of the epicardium and the embryonic myocardial circulation in the mouse. Anat. Rec. Adv. Integr. Anat. Evol. Biol. 1981, 201, 157-168. [CrossRef] [PubMed]

4. Hiruma, T.; Hirakow, R. Epicardial formation in embryonic chick heart: Computer-aided reconstruction, scanning, and transmission electron microscopic studies. Am. J. Anat. 1989, 184, 129-138. [CrossRef]

5. Manner, J.; Pérez-Pomares, J.; Macías, D.; Muñoz-Chápuli, R. The Origin, Formation and Developmental Significance of the Epicardium: A Review. Cells Tissues Organs 2001, 169, 89-103. [CrossRef] [PubMed]

6. Poelmann, R.E.; Groot, A.C.G.-D.; Mentink, M.M.; Bökenkamp, R.; Hogers, B. Development of the cardiac coronary vascular endothelium, studied with antiendothelial antibodies, in chicken-quail chimeras. Circ. Res. 1993, 73, 559-568. [CrossRef]

7. Wessels, A.; Hoff, M.J.V.D.; Adamo, R.F.; Phelps, A.L.; Lockhart, M.M.; Sauls, K.; Briggs, L.E.; Norris, R.A.; van Wijk, B.; Perez-Pomares, J.M.; et al. Epicardially derived fibroblasts preferentially contribute to the parietal leaflets of the atrioventricular valves in the murine heart. Dev. Biol. 2012, 366, 111-124.

8. Yamaguchi, Y.; Cavallero, S.; Patterson, M.; Shen, H.; Xu, J.; Kumar, S.R.; Sucov, H.M. Adipogenesis and epicardial adipose tissue: a novel fate of the epicardium induced by mesenchymal transformation and PPARgamma activation. Proc. Natl. Acad. Sci. USA 2015, 112, 2070-2075. [PubMed]

9. Zangi, L.; Oliveira, M.S.; Ye, L.Y.; Ma, Q.; Sultana, N.; Hadas, Y.; Chepurko, E.; Später, D.; Zhou, B.; Chew, W.L.; et al. Insulin-Like Growth Factor 1 Receptor-Dependent Pathway Drives Epicardial Adipose Tissue Formation After Myocardial Injury. Circulation 2017, 135, 59-72. [CrossRef] [PubMed]

10. Srivastava, D.; Thomas, T.; Lin, Q.; Kirby, M.L.; Brown, D.D.; Olson, E.N. Regulation of cardiac mesodermal and neural crest development by the bHLH transcription factor, dHAND. Nat. Genet. 1997, 16, 154-160. [PubMed]

11. Firulli, A.B.; McFadden, D.G.; Lin, Q.; Srivastava, D.; Olson, E.N. Heart and extra-embryonic mesodermal defects in mouse embryos lacking the bHLH transcription factor Hand1. Nat. Genet. 1998, 18, 266-270. [CrossRef] [PubMed]

12. George, R.M.; Firulli, A.B. Hand Factors in Cardiac Development. Anat. Rec. 2019, 302, 101-107. [CrossRef] [PubMed]

13. Barnes, R.M.; Firulli, B.A.; VanDusen, N.J.; Morikawa, Y.; Conway, S.J.; Cserjesi, P.; Vincentz, J.W.; Firulli, A.B. Hand2 Loss-ofFunction inHand1-Expressing Cells Reveals Distinct Roles in Epicardial and Coronary Vessel Development. Circ. Res. 2011, 108, 940-949. [CrossRef] [PubMed]

14. Zhou, B.; Ma, Q.; Rajagopal, S.K.; Wu, S.M.; Domian, I.J.; Rivera-Feliciano, J.; Jiang, D.; Von Gise, A.; Ikeda, S.; Chien, K.R.; et al. Epicardial progenitors contribute to the cardiomyocyte lineage in the developing heart. Nat. Cell Biol. 2008, 454, 109-113. [CrossRef]

15. Vincentz, J.W.; Casasnovas, J.J.; Barnes, R.M.; Que, J.; Clouthier, D.E.; Wang, J.; Firulli, A.B. Exclusion of Dlx5/6 expression from the distal-most mandibular arches enables BMP-mediated specification of the distal cap. Proc. Natl. Acad. Sci. USA 2016, 113, 7563-7568. [CrossRef]

16. Firulli, B.A.; George, R.M.; Harkin, J.; Toolan, K.P.; Gao, H.; Liu, Y.; Zhang, W.; Field, L.J.; Liu, Y.; Shou, W.; et al. HAND1 loss-offunction within the embryonic myocardium reveals survivable congenital cardiac defects and adult heart failure. Cardiovasc. Res. 2019, 116, 605-618.

17. Vincentz, W.J.; Clouthier, D.E.; Firulli, A.B. Mis-Expression of a Cranial Neural Crest Cell-Specific Gene Program in Cardiac Neural Crest Cells Modulates HAND Factor Expression, Causing Cardiac Outflow Tract Phenotypes. J. Cardiovasc. Dev. Dis. 2020, 7, 13. [CrossRef]

18. Buenrostro, J.D.; Wu, B.; Chang, H.Y.; Greenleaf, W.J. ATAC-seq: A Method for Assaying Chromatin Accessibility Genome-Wide. Curr. Protoc. Mol. Biol. 2015, 109, 21-29. [CrossRef]

19. Kraus, F.; Haenig, B.; Kispert, A. Cloning and expression analysis of the mouse T-box gene Tbx18. Mech. Dev. 2001, 100, 83-86. [CrossRef]

20. Wu, S.-P.; Dong, X.-R.; Regan, J.N.; Su, C.; Majesky, M.W. Tbx18 regulates development of the epicardium and coronary vessels. Dev. Biol. 2013, 383, 307-320.

21. Ratajska, A.; Czarnowska, E.; Ciszek, B. Embryonic development of the proepicardium and coronary vessels. Int. J. Dev. Biol. 2008, 52, 229-236.

22. Vincentz, J.W.; Toolan, K.P.; Zhang, W.; Firulli, A.B. Hand factor ablation causes defective left ventricular chamber development and compromised adult cardiac function. PLoS Genet. 2017, 13, e1006922. [CrossRef] [PubMed]

23. Acharya, A.; Baek, S.T.; Huang, G.; Eskiocak, B.; Goetsch, S.; Sung, C.Y.; Banfi, S.; Sauer, M.F.; Olsen, G.S.; Duffield, J.S.; et al. The bHLH transcription factor Tcf21 is required for lineage-specific EMT of cardiac fibroblast progenitors. Development 2012, 139, 2139-2149. [CrossRef] [PubMed]

24. Lie-Venema, H.; Eralp, I.; Markwald, R.R.; Akker, N.M.S.V.D.; Wijffels, M.C.E.F.; Kolditz, D.P.; Van Der Laarse, A.; Schalij, M.J.; Poelmann, R.E.; Bogers, A.J.J.C.; et al. Periostin expression by epicardium-derived cells is involved in the development of the atrioventricular valves and fibrous heart skeleton. Differentiation 2008, 76, 809-819. [CrossRef] [PubMed] 
25. Vincentz, J.W.; VanDusen, N.J.; Fleming, A.B.; Rubart, M.; Firulli, B.A.; Howard, M.J.; Firulli, A.B. A Phox2- and Hand2-Dependent Hand1 cis-Regulatory Element Reveals a Unique Gene Dosage Requirement for Hand2 during Sympathetic Neurogenesis. J. Neurosci. 2012, 32, 2110-2120. [CrossRef]

26. Barnes, R.M.; Firulli, B.A.; Conway, S.J.; Vincentz, J.W.; Firulli, A.B. Analysis of the Hand1 cell lineage reveals novel contributions to cardiovascular, neural crest, extra-embryonic, and lateral mesoderm derivatives. Dev. Dyn. 2010, $239,3086-3097$.

27. Balmer, G.M.; Bollini, S.; Dubé, K.N.; Martinez-Barbera, J.P.; Williams, O.; Riley, P.R. Dynamic haematopoietic cell contribution to the developing and adult epicardium. Nat. Commun. 2014, 5, 4054. [CrossRef]

28. Firulli, B.A.; Howard, M.J.; McDaid, J.R.; McIlreavey, L.; Dionne, K.M.; E Centonze, V.; Cserjesi, P.; Virshup, D.M.; Firulli, A.B. PKA, PKC, and the Protein Phosphatase 2A Influence HAND Factor Function. Mol. Cell 2003, 12, 1225-1237. [CrossRef]

29. Firulli, B.A.; Krawchuk, D.; Centonze, V.E.; Virshup, D.M.; Conway, S.J.; Cserjesi, P.; Laufer, E.; Firulli, A.B. Altered Twist1 and Hand2 dimerization is associated with Saethre-Chotzen syndrome and limb abnormalities. Nat. Genet. 2005, 37, 373-381. [CrossRef] [PubMed]

30. Firulli, B.A.; Redick, B.A.; Conway, S.J.; Firulli, A.B. Mutations within Helix I of Twist1 Result in Distinct Limb Defects and Variation of DNA Binding Affinities. J. Biol. Chem. 2007, 282, 27536-27546. [CrossRef]

31. George, M.R.; Duan, Q.; Nagle, A.; Kathiriya, I.S.; Huang, Y.; Rao, K.; Haldar, S.M.; Bruneau, B.G. Minimal in vivo requirements for developmentally regulated cardiac long intergenic non-coding RNAs. Development 2019, 146, dev185314. [CrossRef] [PubMed] 